\title{
Incidence of injuries and other health problems in the Auckland Citibank marathon, 1993
}

\author{
Peter Satterthwaite, Peter Larmer, James Gardiner, Robyn Norton
}

\begin{abstract}
Objective-To describe the incidence of injuries and other health problems sustained during participation in a marathon.

Methods-A cohort study was undertaken involving the 1993 Auckland Citibank marathon participants. Demographic data and information on injuries and other health problems sustained during, immediately after, and $7 \mathrm{~d}$ following the marathon were obtained from a pre-race questionnaire, the medical aid posts, and a post-race questionnaire.
\end{abstract}

Results-Of the 1219 starters, 916 (75.1\%) completed both questionnaires. Seventy five individuals $(6.2 \%)$ sought assistance at the medical aid posts. During or immediately after the marathon, 283 systemic health problems were reported by 218 respondents $(23.8 \%)$ and 2671 specific health problems were reported by 846 respondents $(92.4 \%)$. In the $7 \mathrm{~d}$ following the marathon, 1905 specific health problems were reported by 723 respondents $(79.2 \%)$. The majority of the specific health problems were blisters, stiffness, and pain, predominantly involving the lower limbs.

Conclusions-Although a high proportion of participants experienced health problem during the race, very few of these problems were serious. Many of the entrants were still experiencing problems $7 \mathrm{~d}$ after the marathon.

(Br F Sports Med 1996;30:324-326)

Key terms: marathon; epidemiology; injuries; health problems

Injury Prevention Research Centre, Department of Community Health, University of Auckland, Auckland, New Zealand P Satterthwaite P Larmer J Gardiner R Norton

Correspondence to: Dr Peter Satterthwaite, Injury Prevention Research Centre, Department of Community Health, University of Auckland, Auckland, New Zealand.

Accepted for publication 9 July 1996
Regular, moderate intensity physical activity has been shown to have both physical and mental health benefits, such that the Centers for Disease Control and Prevention and the American College of Sports Medicine have recently recommended that every US adult should accumulate 30 minutes or more of moderate intensity physical activity on most, preferably all, days of the week. ${ }^{1}$ However, the short term benefits and acute adverse consequences associated with more intensive physical activity have not been well documented.

Review of published reports on injuries and other health problems associated with running, and in particular with marathons, indicates that very few well designed epidemiological studies have been conducted. ${ }^{2-4}$ This paper describes the incidence of injuries and other health problems occurring during, immediately after, and seven days following a marathon.

\section{Methods}

All individuals planning to participate in the 1993 Auckland Citibank marathon (entrants) completed a preregistration form. This form sought information on the individual's name, age, gender, previous best marathon time, and goal time for the Auckland marathon. Unique race numbers were assigned by race organisers to each entrant. Individuals were then requested to register and collect their race packs during the two days preceding the marathon. At registration, each entrant was invited to participate in the study, to sign a consent form agreeing to their participation, and to complete an interviewer administered pre-race questionnaire.

During the race, information was collected on all injuries and other health problems sustained by runners who attended the medical aid posts. Medical aid posts were placed along the race as well as at the finish line. In the week following the race, all entrants were mailed a post-race questionnaire. In addition, a pre-race questionnaire and consent form was sent to those entrants who had not originally completed it. The post-race questionnaire inquired about injuries and other medical problems sustained both during or immediately after the marathon and in the seven days following the marathon. Participants were asked about any systemic health problems, such as light headedness, nausea, diarrhoea, stomach problems, and chills. They were then asked about specific health problems, using a matrix of 13 body sites by 11 problem types. The entrants were asked to complete an identical matrix for problems occurring in the seven days after the marathon. A stamped addressed envelope was provided for the return of the questionnaire(s). Those not returning the post-race questionnaire within two weeks were sent another mailing. Residual non-responders were then contacted by telephone and asked either to return the completed questionnaire or to complete the questionnaire by telephone at that time.

Data entry and analysis were undertaken using Epi Info version 5. This study had the approval of the University of Auckland human subjects ethics committee.

\section{Results}

The race was run in near perfect conditions with minimum and maximum temperatures of 11.9 and $17.2^{\circ} \mathrm{C}$, with an average humidity of 


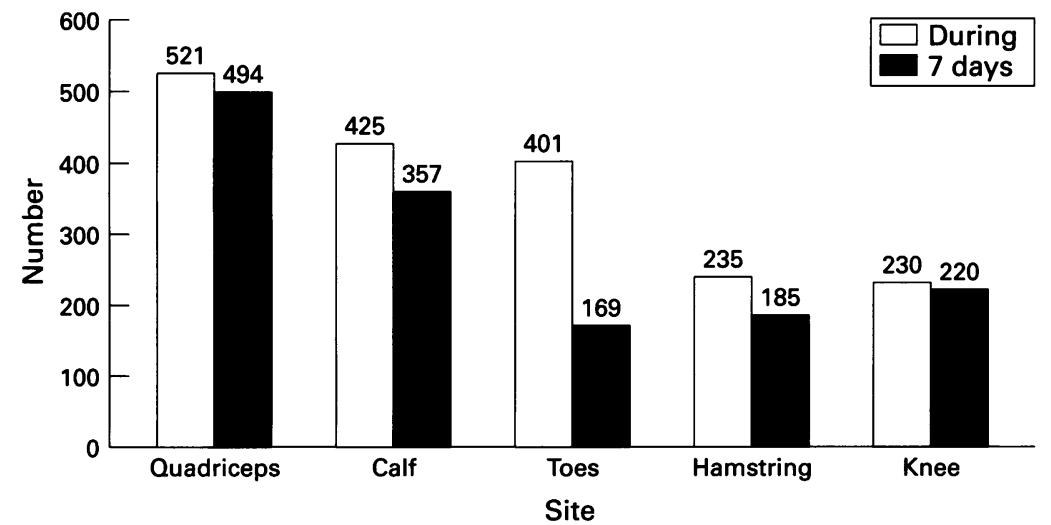

Figure 1 Reported injuries by body site, during, immediately after, and $7 d$ after the race.

$63 \%$. Of the 1357 entrants, $1219(89.8 \%)$ are known to have started, 120 did not start, and the status of the remaining 18 is unknown ( 10 of whom were overseas entrants). Of those who are known to have started, $1185(97.2 \%)$ finished, 31 did not finish, and the status of three is unknown.

The number of known starters who responded to the pre-race questionnaire was 1054 , a response rate of $86.5 \%$. The number of starters who also responded to the post-race questionnaire was 916, a response rate of $75.1 \%$. A comparison of the age, gender and finish times of starters who completed both surveys compared with those who did not showed no significant differences $(P>0.05)$.

Males made up $80.3 \%$ and females $19.7 \%$ of responding starters. The average age of these individuals was 38.6 years with a range of 19 to 74 years and a standard deviation of 9.8 years. The age distribution was skewed towards the right, indicating that there were relatively more older runners.

\section{MEDICAL AID POST HEALTH PROBLEMS}

The total number of runners who sought assistance at the medical aid posts was 75 , $6.2 \%$ of the known starters. Of these, $74.6 \%$ were male, and $25.4 \%$ female. The average age of those who attended the medical aid post was 36.6 years. Only one starter was referred to hospital. The injuries and other health problems seen at the medical aid post were cramps $(19.7 \%)$, exhaustion $(14.5 \%)$, haematomas

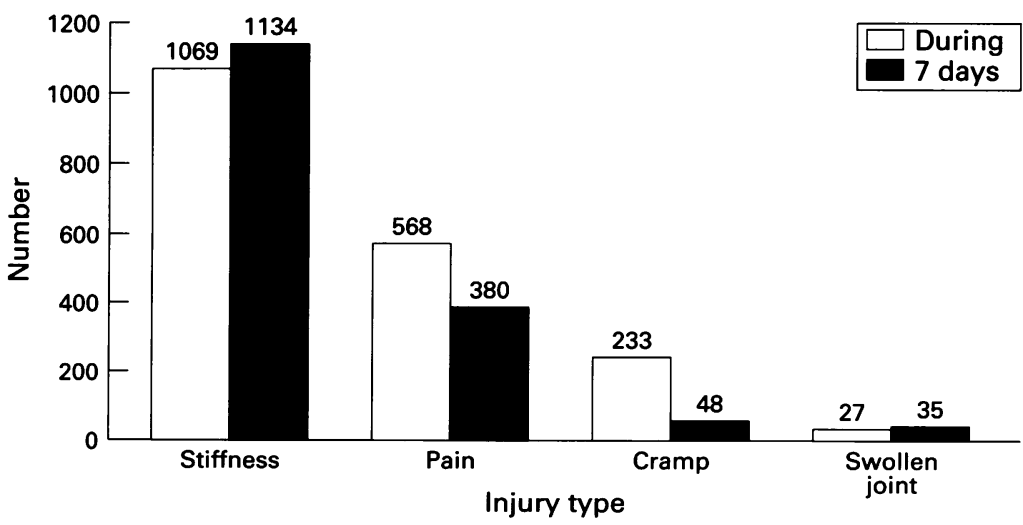

Figure 2 Reported injuries by type of injury, during, immediately after, and $7 d$ after the race.
$(14.5 \%)$, blisters $(14.5 \%)$, light headedness $(6.6 \%)$, tendonitis $(6.6 \%)$, and other $(23.7 \%)$.

OTHER HEALTH PROBLEMS DURING OR

IMMEDIATELY AFTER

There were 283 systemic health problems reported during or immediately after the marathon by 218 respondents $(23.8 \%)$. Men comprised $73.9 \%$ and women $26.1 \%$ of those who reported problems. The average age of those who had problems was 36.1 years. The most common types of problem reported were: lightheadness $(27.8 \%)$, nausea $(16.9 \%)$, stomach upsets $(13.9 \%)$, chills $(12.3 \%)$, headache $(10.8 \%)$, and diarrhoea $(10.8 \%)$.

There were 2671 specific health problems reported during or immediately after the marathon by 846 respondents $(92.4 \%)$, an average of 2.9 problems per respondent who reported problems. Men comprised $80.1 \%$ and women $19.9 \%$ of those who reported problems. The average age of those who had problems was 38.2 years. The most common body sites involved were: feet/toes (22.6\%), quadriceps $(19.8 \%)$, calf $(16.0 \%)$, hamstring $(9.1 \%)$, and knee $(8.8 \%)$ (fig 1 ). The most common types of problems reported were: stiffness $(40.0 \%)$, pain $(21.3 \%)$, and blisters (14.9\%) (fig 2).

OTHER HEALTH PROBLEMS 7 DAYS AFTER

There were 1905 specific health problems reported in the $7 \mathrm{~d}$ following the marathon by 723 respondents $(79.2 \%)$, an average of 2.1 per respondent who reported problems. Men comprised $80.2 \%$ and women $19.8 \%$ of those who reported problems. The average age of those who had problems was 37.8 years. The most common body sites involved were: quadriceps $(28.2 \%)$, calf $(20.5 \%)$, feet/toes $(14.8 \%)$, knee $(12.7 \%)$, and hamstrings $(9.9 \%)$ (fig 1 ). The most common types of problems reported were: stiffness $(60.1 \%)$, pain $(20.2 \%)$, and blisters $(7.6 \%)$ (fig 2 ).

\section{Discussion}

In this paper we describe the injuries and other health problems sustained by runners in a large international marathon. Over $90 \%$ of the respondents reported health problems during or immediately after the race. However, very few of these problems were serious, with no fatalities, one hospital admission, and only $6 \%$ requiring immediate medical attention. In the week following the race, almost $80 \%$ reported health problems, although again few of these problems were serious. Not surprisingly, the vast majority of problems sustained both during and after the race were associated with the lower limbs.

These findings are likely to provide a more unbiased estimate of the true incidence and patterns of health problems associated with running a marathon than has been available previously. Not only was the response rate to the post-race questionnaire considerably higher than that reported in previous similar studies $^{23}$ but also the gender, age, and finish times of non-responders were comparable with responders. In addition, the finding that about 
$90 \%$ of responders sustained health problems is almost identical to that reported by Kretsch et al in the 1980 Melbourne marathon. ${ }^{4}$

The finding that almost all individuals sustained health problems, albeit minor, suggests that such problems may be inevitable given the nature of the activity. In particular, as marathon running is an acute endurance event involving the lower limbs, it is perhaps not surprising that the most common types of problem reported were blisters, stiffness, and pain affecting predominantly the lower limbs. Of greater concern may be the proportions reporting systemic health problems during or immediately after the race. As many of these problems could be linked to symptoms of mild dehydration, if the weather conditions were less favourable in future years this might lead to more serious health problems.

Given that the proportion of individuals attending the medical aid posts was very small in comparison with the proportions who reported a health problem during the race, the use of such data to describe the incidence and patterns of injuries associated with marathons is clearly inappropriate.

In summary, most of the athletes who participated in the 1993 Auckland marathon experienced health problems as a result. Very few of these problems were serious and none was fatal. Only a small proportion required immediate medical attention. However, the significant proportion of individuals who reported systemic problems is of some concern. Marathon participants, race organisers, and attending medical staff should be made aware of the health problems that are likely to be sustained as a result of running a marathon, as identified in this study. In addition, the importance of rehydration and the provision of adequate rehydration facilities during a marathon should continue to be highlighted.

The assistance of the Auckland Citibank Marathon organisers, volunteers, and participants is acknowledged. In addition we wish to acknowledge Dr Henare Broughton, medical director for the 1992 Auckland Marathon, for his support in undertaking the project. The Injury Prevention Research Centre is jointly funded by the Health Research Council of New Zealand and the Accident Rehabilitation and Compensation Insurance Corporation.

1 Pate RR, Pratt M, Blair SN, Haskell W, Macera C, Bouchard C. Physical activity and public health. $\mathscr{f} A M A$ 1995;273:402-7.

2 Ridley S A, Rogers PN, Wright IH. Glasgow marathons 982-7: a review of the medical problems. Scot Med $\mathscr{I}$ 1990;35:9-11.

3 Nicholl JP, Williams BT. Medical problems before and after a popular marathon. $B M \mathcal{F} 1982 ; 285: 1465-6$.

4 Kretsch A, Grogan R, Duras P, Allen F, Sumner J, Gillam I. 1980 Melbourne marathon study. Med f Aust 1984;141:
rretsch A, Grogan R, Duras P, Allen F, Sumner J, Gillam I. 809-14. 